\title{
ASYMPTOTIC SOLUTION OF DIFFERENTIAL EQUATIONS IN A DOMAIN CONTAINING A REGULAR SINGULAR POINT
}

\author{
N. D. KAZARINOFF and R. McKELVEY
}

1. Introduction. In this paper we study the asymptotic behavior in $\lambda$ of the solutions about the origin in the $z$-plane of the differential equation

$$
z^{2} \frac{d^{2} u}{d z^{2}}-\lambda^{2} P(z, \lambda) u=0 .
$$

Both the variable $z$ and the parameter $\lambda$ are complex. The coefficient $P(z, \lambda)$ is assumed to be analytic and single-valued in $\lambda$ at infinity and in $z$ throughout a bounded, closed, simply connected domain $D$ containing $z=0$. The important restriction is imposed ${ }^{1}$ that $P(z, \lambda) \neq 0$ for $z \in D$ when $|\lambda|>N$.

Asymptotic series in descending powers of $\lambda$ are obtained, which are formal solutions of the differential equation, and it is shown that their partial sums are asymptotic representations for the solutions of this equation. The coefficients in these series are determined recursively in terms of the Taylor series of $P(z, \lambda)$ about $\lambda=\infty$. It is to be noted that the first term of each series may be found from results of Cashwell (3), who considered the differential equation in a somewhat different form. We remark that the equation which we are considering is exceptional in the theory of Evans (4), and cannot be treated by his procedure.

As an application of the general method, we derive an asymptotic expansion of the Whittaker function $M_{2 a m+b, m}(z)$ for numerically large $m, \mathscr{R}(m) \geqslant 0$, and $a$ and $b$ bounded.

2. Formal solutions. By a change of notation we write the differential equation as

$$
L[u] \equiv \frac{d^{2} u}{d z^{2}}-\left\{\lambda^{2} \frac{\phi^{2}(z)}{z^{2}}+\lambda \frac{\tau(\lambda)}{z^{2}}+\lambda \frac{\theta(z, \lambda)}{z}\right\} u=0
$$

with

$$
\theta(z, \lambda)=\sum_{0}^{\infty} \theta_{j}(z) / \lambda^{j}
$$

The functions $\theta_{j}(z)$ and $\phi(z)$ are analytic in $D$, and $\phi(z)$ has no zeros there. By the transfer of a constant to $\lambda^{2}$, we adopt the normalization $\phi(0)=1$.

Received March 9, 1955.

'The constant $N$ is to be chosen so large that it satisfies all subsequent requirements to be placed upon it. 
Thus the function

$$
\omega(z)=\frac{\phi(z)-1}{z}, z \neq 0, \omega(0)=\phi^{\prime}(0),
$$

is analytic. Lastly we define

$$
\Omega(z)=\int_{0}^{z} \omega(t) d t .
$$

The exponents of the equation $L[u]=0$ about $z=0$ are $\frac{1}{2} \pm \mu(\lambda)$, where

$$
\mu(\lambda)=\lambda\left\{1+\frac{\tau(\lambda)}{\lambda}+\frac{1}{4 \lambda^{2}}\right\}^{\frac{1}{2}} .
$$

It is convenient to introduce the notation that

$$
\mu(\lambda)-\lambda=\kappa(\lambda), \kappa(\lambda)=\sum_{0}^{\infty} \kappa_{j} / \lambda^{j} .
$$

We now choose $n$ to be a non-negative integer which will remain fixed for the remainder of the discussion. In order to find formal solutions of $L[u]=0$, let us consider the functions $y_{ \pm n}(z, \lambda)$ defined by the relations

$$
y_{ \pm n}(z, \lambda)=z^{\frac{1}{2 \pm \mu(\lambda)}} e^{ \pm \lambda \Omega(z)} A_{ \pm n}(z, \lambda) .
$$

Here the functions $A_{ \pm n}(z, \lambda)$ are certain polynomials in $1 / \lambda$ with coefficients analytic in $z$, i.e.,

$$
A_{ \pm n}(z, \lambda)=\sum_{0}^{n} \alpha_{ \pm j}(z) / \lambda^{j}
$$

By direct computation

with

$$
L\left[y_{ \pm n}\right]=z^{-\frac{1}{2} \pm \mu(\lambda)} e^{ \pm \lambda \Omega(z)} \frac{H_{ \pm n}(z, \lambda)}{\lambda^{n}},
$$

$$
\begin{aligned}
H_{ \pm n}(z, \lambda)=\lambda^{n}\left[z A_{n}^{\prime \prime}(z, \lambda)+\{1 \pm 2[\lambda \phi(z)+\kappa(\lambda)]\} A_{ \pm n}^{\prime}(z, \lambda)\right. \\
\left.+\lambda\left\{2 \kappa(\lambda) \omega(z)-\theta(z, \lambda) \pm \phi^{\prime}(z)\right\} A_{ \pm n}(z, \lambda)\right] .
\end{aligned}
$$

The primes denote differentiation with respect to $z$.

Expanding the functions $H_{ \pm n}(z, \lambda)$ in powers of $\lambda$ and equating the coefficients of $\lambda^{n+1}, \lambda^{n}, \ldots, \lambda$ to zero, one obtains a recursive set of first order linear differential equations in the functions $\alpha_{ \pm j}(z)$. These equations are satisfied, and hence the functions $H_{ \pm n}(z, \lambda)$ are bounded provided that

where

$$
\begin{aligned}
\alpha_{ \pm 0}(z) & =\phi^{-\frac{1}{z}} \exp \left(\mp \int_{0}^{z}\left\{2 \kappa_{0} \omega(z)-\theta_{0}(z)\right\} \frac{d t}{2 \phi(t)}\right), \\
\alpha_{ \pm m}(z) & =\alpha_{ \pm 0}(z) \int_{0}^{z} \frac{F_{ \pm m}(t)}{2 \phi(t) \alpha_{ \pm 0}(t)} d t, \quad m=1,2, \ldots, n,
\end{aligned}
$$

$$
\begin{aligned}
F_{ \pm m}(z)=-2 \sum_{j=0}^{m-1} \kappa_{j} \alpha_{ \pm(m-j-1)}(z) & \\
& \mp\left\{\left[z \alpha_{ \pm(m-1)}^{\prime}(z)\right]^{\prime}+\sum_{j=1}^{m}\left[2 \kappa_{j} \omega(z)-\theta_{j}(z)\right] \alpha_{ \pm(m-j)}(z)\right\} .
\end{aligned}
$$


The coefficients $\alpha_{ \pm j}(z)$ so defined are analytic; and $\alpha_{ \pm 0}(0)=1, \alpha_{ \pm m}(0)=0$, $m=1,2, \ldots, n$. Moreover, $H_{ \pm n}(z, \lambda)$ and $A_{ \pm n}(z, \lambda)$ are also analytic for $z \in D$ and $|\lambda|>N$.

If in the above discussion the integer $n$ were to be replaced by $\infty$, one would have the result that $L\left[y_{ \pm \infty}\right]=0$. Formally, then, the infinite series would satisfy the given differential equation. Actually, of course, they might be divergent.

It is to be noted that the above procedure is closely analogous to that employed in (1) near an ordinary point of the equation. The modifications relate directly to the presence of the pole.

3. A related differential equation. The Wronskian of the functions $y_{+n}(z, \lambda)$ and $y_{-n}(z, \lambda)$,

$$
W(z, \lambda) \equiv y_{+n}(z, \lambda) y_{-n}^{\prime}(z, \lambda)-y_{+n}^{\prime}(z, \lambda) y_{-n}(z, \lambda),
$$

is, by simple computation,

$$
W(z, \lambda)=\left|\begin{array}{ll}
A_{+n} & A_{-n} \\
z A_{+n}^{\prime}+\left[\frac{1}{2}+\lambda \phi+\kappa\right] A_{+n} & z A_{-n}^{\prime}+\left[\frac{1}{2}-(\lambda \phi+\kappa)\right] A_{-n}
\end{array}\right| .
$$

From this relation we see that $W(z, \lambda)$ is analytic for $z \in D$ and $|\lambda|>N$ and that its value at $z=0$ is $-2 \mu(\lambda)$. Differentiating both sides of (4) and replacing the functions $y_{ \pm n}{ }^{\prime \prime}(z, \lambda)$ by their equivalents obtained from (3), one finds that

$$
W^{\prime}(z, \lambda)=\frac{1}{\lambda^{n}}\left\{A_{+n} H_{-n}-A_{-n} H_{+n}\right\} .
$$

Since $W(0, \lambda)=-2 \mu(\lambda)$, by integrating this expression from zero to $z$, we may conclude that

$$
W(z, \lambda)=-2 \mu(\lambda)+z B(z, \lambda) / \lambda^{n} .
$$

Here $B(z, \lambda)$ designates a function which is analytic and hence bounded for $z \in D$ and $|\lambda|>N$. For future use, we adopt $B(z, \lambda)$ as generic notation for such a function.

It is familiar that the linearly independent functions $y_{+n}(z, \lambda)$ and $y_{-n}(z, \lambda)$ satisfy the second order differential equation

$$
\frac{d^{2} y}{d z^{2}}-\frac{W^{\prime}}{W} \frac{d y}{d z}-\left\{\frac{y_{+n}^{\prime \prime} y_{-n}^{\prime}-y_{+n}^{\prime} y_{-n}^{\prime \prime}}{W}\right\} y=0 .
$$

Again evaluating $y_{ \pm n}{ }^{\prime \prime}(z, \lambda)$ by means of $(3)$, the bracketed quantity is found to be

$$
\frac{\lambda^{2} P(z, \lambda)}{z^{2}}+\frac{G(z, \lambda)}{z \lambda^{n}}
$$

where

$$
G(z, \lambda)=\frac{1}{W}\left|\begin{array}{ll}
H_{+n} & H_{-n} \\
z A_{+n}^{\prime}+\left[\frac{1}{2}+\lambda \phi+\kappa\right] A_{+n} & z A_{-n}^{\prime}+\left[\frac{1}{2}-(\lambda \phi+\kappa)\right] A_{-n}
\end{array}\right| .
$$


The transformation of variable

$$
v(z, \lambda)=\left[\frac{-2 \mu(\lambda)}{W(z, \lambda)}\right]^{\frac{1}{2}} y(z, \lambda)
$$

carries the differential equation (6) into

$$
\frac{d^{2} v}{d z^{2}}-\left\{\frac{\lambda^{2} P(z, \lambda)}{z^{2}}+\frac{B(z, \lambda)}{z \lambda^{n}}\right\} v=0 .
$$

This equation is in an evident sense an $n$th degree approximation to the given differential equation (1). The solutions $v_{ \pm n}(z, \lambda)$ of $(8)$ which correspond to the solutions $y_{ \pm n}(z, \lambda)$ of (6) by the transformation (7) are, from (5), of the form

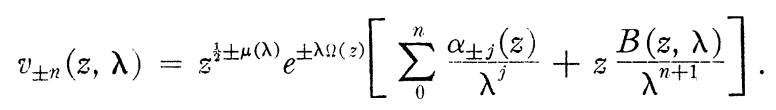

These functions are linearly independent. Their Wronsisian, which is constant as to $z$, has the value $-2 \mu(\lambda)$.

4. Comparison of $v_{+n}(z, \lambda)$ with solutions of $L[u]=0$. We introduce the function

$$
\sigma(z)=z e^{\Omega(z)} .
$$

Clearly, $\sigma(z)$ is schlicht (3). Let us confine our attention to a region $E \subset D$ whose one-to-one image, $\mathscr{E}$, in the $\sigma$-plane is a circle centered at the origin. For definiteness we restrict the argument of $z$ to be single-valued, making a cut along the negative real axis in $\mathscr{E}$.

By familiar use of the method of variation of parameters, the differential equation $L[u]=0$ may be shown to be equivalent to an integral equation of the form

$$
\begin{array}{r}
u(z, \lambda)=v(z, \lambda)+\frac{1}{\lambda^{n+1}} \int_{z_{o}}^{z}\left\{v_{+n}(z, \lambda) v_{-n}(t, \lambda)-v_{+n}(t, \lambda) v_{-n}(z, \lambda)\right\} \\
\cdot \frac{B(t, \lambda)}{t} u(t, \lambda) d t,
\end{array}
$$

where $v(z, \lambda)$ is any solution of $(8)$ and $z_{0}$ is any point of $E$ (including $z_{0}=0$, provided the integral is convergent).

If the $\sigma$-plane is subdivided into four quadrants, it is possible to choose $z_{0}$ and $v(z, \lambda)$ in equation (9) so that the integral in this equation may be estimated and asymptotic forms for two linearly independent solutions of (9) derived in each quadrant. For illustrative purposes, a discussion of the derivation of the asymptotic forms of two of these solutions is given below.

(a) Solutions subdominant in $\lambda$ for small $z$. We suppose for the moment that $\mathscr{R}(\mu) \geqslant 0$. In (9) choose $v(z, \lambda)=v_{+n}(z, \lambda), z_{0}=0$, and the path of integration

2In the sense employed, for example, by Langer (6). 
the image of a radius vector in $\mathscr{E}$. We call the solution of (9) which is thus determined $u_{s}(z, \lambda)$. It will be evident that this solution is well defined for all $z$ in $E$. Introducing the notation

$$
\begin{gathered}
V_{ \pm n}(z, \lambda)=z^{-\frac{1}{2} \mp \mu(\lambda)} e^{\mp \lambda \Omega(z)} v_{ \pm n}(z, \lambda), \\
U_{s}(z, \lambda)=z^{-\frac{1}{2}-\mu(\lambda)} e^{-\lambda \Omega(z)} u_{s}(z, \lambda),
\end{gathered}
$$

we may rewrite equation (9) as

$$
U_{s}(z, \lambda)=V_{+n}(z, \lambda)+\frac{1}{\lambda^{n+1}} \int_{0}^{z} K_{s}(z, t, \lambda) U_{s}(t, \lambda) d t
$$

where

$$
\begin{array}{r}
K_{s}(z, t, \lambda)=\left\{V_{+n}(z, \lambda) V_{-n}(t, \lambda)-V_{+n}(t, \lambda) V_{-n}(z, \lambda)\right. \\
\left.\left[\frac{\sigma(t)}{\sigma(z)}\right]^{2 \mu} e^{-2 \kappa[\Omega(t)--\Omega(z)]}\right\} B(t, \lambda) .
\end{array}
$$

The functions $V_{ \pm n}(z, \lambda), B(z, \lambda)$, and the exponential occurring in $K_{s}(z, t, \lambda)$ are bounded for $z \in E$ and $|\lambda|>N$. The quantity $[\sigma(t) / \sigma(z)]^{2 \mu}$ is bounded on the contour of integration in (10) since $|\sigma(t)| \leqslant|\sigma(z)|$ on the contour and $\mathscr{R}(\mu) \geqslant 0$. Thus $K_{s}(z, t, \lambda)$ is bounded on the chosen contour which is of finite length. In fact, there is a uniform bound to the lengths of the contours corresponding to each $z \in E$. It follows that

$$
\int_{0}^{z}\left|K_{\underline{s}}(z, t, \lambda)\right| \cdot|d t|
$$

is also bounded for $z \in E$. As a consequence: ${ }^{3}$

$$
U_{s}(z, \lambda)=V_{+n}(z, \lambda)+\frac{B(z, \lambda)}{\lambda^{n+1}} .
$$

Thus

$$
u_{s}(z, \lambda)=z^{\frac{1}{2}+\mu(\lambda)} e^{\lambda \Omega(z)}\left\{\sum_{0}^{n} \frac{\alpha_{j}(z)}{\lambda^{j}}+\frac{B(z, \lambda)}{\lambda^{n+1}}\right\},
$$

for $z \in E$ and $|\lambda|>N$.

An analogous discussion can be given when $\mathscr{R}(\mu) \leqslant 0$. Inasmuch as this is the case, we have established the following theorem.

Theorem 1. Over each half plane $\mathscr{R}( \pm \mu) \geqslant 0$, the differential equation $L[u]=0$ admits of a solution $u_{ \pm s}(z, \lambda)$ for $z \in E$ and $|\lambda|>N$, where

$$
u_{ \pm s}(z, \lambda)=z^{\frac{3}{2} \pm \mu(\lambda)} e^{ \pm \lambda \Omega(z)}\left\{\sum_{0}^{n} \frac{\alpha_{ \pm j}(z)}{\lambda^{j}}+\frac{B(z, \lambda)}{\lambda^{n+1}}\right\} .
$$

The functions $\mu(\lambda), \Omega(z)$, and $\alpha_{ \pm j}(z)$ are all defined in $\S 2$.

\footnotetext{
${ }^{3}$ See Lemma of Langer (5, p. 479). The extension to a complex variable involves no difficulty.
} 
(b) Solutions dominant in $\lambda$ for small $z$. We now momentarily suppose that $\mathscr{R}(\mu) \geqslant 0$ and $\mathscr{I}(\mu) \leqslant 0$. In $(9)$ set $v(z, \lambda)=v_{-n}(z, \lambda)$. The contour of integration is to be the curve whose image in $\mathscr{E}$ begins at the point of the boundary of the circle at which $\arg (\sigma)=\pi$ and is composed of an arc of this circle together with a radial line segment terminating at $\sigma(z)$. As we are considering values of $\mu(\lambda)$ in the fourth quadrant, we call the solution of (9) determined by these conditions $u_{4}(z, \lambda)$. If we write,

$$
U_{4}(z, \lambda)=z^{-\frac{1}{2}+\mu(\lambda)} e^{\lambda \Omega(z)} u_{4}(z, \lambda),
$$

then equation (9) may be written in the form

$$
U_{4}(z, \lambda)=V_{-n}(z, \lambda)+\lambda^{-n-1} \int_{z_{0}}^{z} K_{4}(z, t, \lambda) U_{4}(t, \lambda) d t,
$$

where

$$
\begin{aligned}
K_{4}(z, t, \lambda)=\left\{V_{+n}(z, \lambda) V_{-n}(t, \lambda)\left[\frac{\sigma(t)}{\sigma(z)}\right]^{-2 \mu}\right. & e^{2 \kappa[\Omega(t)-\Omega(z)]} \\
& \left.-V_{+n}(t, \lambda) V_{-n}(z, \lambda)\right\} B(t, \lambda) .
\end{aligned}
$$

On the present contour both $|\sigma(z)| \leqslant|\sigma(t)|$ and $\arg \sigma(z) \leqslant \arg \sigma(t)$; hence, $[\sigma(t) / \sigma(z)]^{-2 \mu}$ is bounded on the contour. Reasoning as before, we may conclude that

$$
U_{4}(z, \lambda)=V_{-n}(z, \lambda)+\frac{B(z, \lambda)}{\lambda^{n+1}}
$$

for all $z \in E$ and $|\lambda|>N$. Thus when $z \neq 0$,

$$
u_{4}(z, \lambda)=z^{\frac{1}{2}-\mu(\lambda)} e^{-\lambda \Omega(z)}\left\{\sum_{0}^{n} \frac{\alpha_{-j}(z)}{\lambda^{j}}+\frac{B(z, \lambda)}{\lambda^{n+1}}\right\} .
$$

If $\mathscr{R}(\mu)=0$, the above relation holds for all $z \in E$.

The results of the preceding analysis are included and extended in the following theorem.

THeorem 2. Over each quadrant $(\nu-1) \pi / 2 \leqslant \arg (\mu) \leqslant \frac{1}{2} \nu \pi$, the differential equation $L[u]=0$ admits of a solution $u_{v}(z, \lambda)$ for all $z \in E$ except $z=0$ and for $|\lambda|>N$, where

$$
\begin{array}{ll}
u_{\nu}(z, \lambda)=z^{\frac{1}{2}-\mu(\lambda)} e^{-\lambda \Omega(z)}\left\{\sum_{0}^{n} \frac{\alpha_{-j}(z)}{\lambda^{j}}+\frac{B(z, \lambda)}{\lambda^{n+1}}\right\}, & \nu=1 \text { and } 4, \\
u_{v}(z, \lambda)=z^{\frac{1}{2}+\mu(\lambda)} e^{\lambda \Omega(z)}\left\{\sum_{0}^{n} \frac{\alpha_{+j}(z)}{\lambda^{j}}+\frac{B(z, \lambda)}{\lambda^{n+1}}\right\}, & \nu=2 \text { and } 3 .
\end{array}
$$

When $\mathscr{R}(\mu)=0$, the conclusions of the Theorem may be drawn even when $z=0$. When $2 \mu(\lambda)$ is integral, the logarithmic term possibly occurring in $u_{\nu}(z, \lambda)$ is of the form $c(\lambda) \ln z u_{ \pm s}(z, \lambda)$, according as $\nu=1,4$ or $\nu=2,3$, and is subsumed in the undetermined function $B(z, \lambda) / \lambda^{n+1}$. The connection between the above results and the Fuchsian theory is manifest. 
5. An application. Among the special functions which satisfy differential equations of the type $L[u]=0$ are the Whittaker functions commonly denoted $M_{k, m}(z)$. They are solutions of the differential equation (2)

$$
\frac{d^{2} w}{d z^{2}}+\left(-\frac{1}{4}+\frac{k}{z}+\frac{\frac{1}{4}-m^{2}}{z^{2}}\right) W=0,
$$

The parameters and variable $z$ are taken to be complex. We consider the function $M_{k, m}(z)$ for $|m|$ and $|k|$ large subject to the restrictions that $|z|$ be bounded, that $k=2 a m+b$, where the secondary parameters $a$ and $b$ vary boundedly, and that $\mathscr{R}(m) \geqslant 0$. For the structure of $M_{k, m}(z)$ when $|z|$ and $|k|$ are bounded but $|m|$ is large see (2, p. 93).

If we make the identification

$$
\frac{\lambda^{2} P(z, \lambda)}{z^{2}}=\frac{m^{2}}{z^{2}}\left(1-\frac{2 a z}{m}+\frac{\frac{1}{4} z^{2}-b z-\frac{1}{4}}{m^{2}}\right),
$$

a simple computation yields the results that for equation (11)

$$
y_{ \pm 1}(z, m)=z^{\frac{1}{2} \pm m} e^{ \pm a z}\left[1 \pm \frac{z}{2 m}\left(\frac{z}{8}-b \pm a-\frac{a^{2} z}{2}\right)\right] .
$$

A calculation of $y_{ \pm n}(z, m)$ for an $n$ greater than one is feasible as the quantities $\alpha_{ \pm n}(z)$ may be seen to involve only polynomials in $z$, the secondary parameters $a$ and $b$, and $e^{ \pm a z}$. The hypotheses of the theory just presented are easily seen to be satisfied by equation (11) for $z$ in any circle with center at the origin provided that $|m|$ is sufficiently large. Further, the bound implied in $B(z, m)$ will not depend upon $a$ and $b$ if $|a|$ and $|b|<M$.

It remains to identify $M_{k, m}(z)$ as a linear combination of the solutions given by the theorems of the last section. Since $M_{k, m}(z)$ and $u_{s}(z, m)$ are both subdominant for $\mathscr{R}(m) \geqslant 0$ and since

$$
\lim _{z \rightarrow 0} z^{-\frac{1}{2}-m} M_{k, m}(z)=1, \quad \lim _{z \rightarrow 0} z^{-\frac{1}{2}-m} u_{s}(z, m)=1,
$$

it follows that

Thus,

$$
M_{k, m}(z)=u_{s}(z, m)
$$

THEOREM 3.

$$
M_{k, m}(z)=z^{\frac{1}{2}+m} e^{-a z}\left[1+\frac{z}{2 m}\left(\frac{z}{8}-b+a-\frac{a^{2} z}{2}\right)+\frac{B(z, m)}{m^{2}}\right],
$$

when $\mathscr{R}(m) \geqslant 0, k=2 a m+b$, and $a, b$, and $z$ are bounded.

\section{REFERENCES}

1. G. D. Birkhoff, On the asymptotic character of the solutions of certain differential equations containing a parameter, Trans. Amer. Math. Soc., 9 (1908), 214-231. 
2. H. Buchholz, Die konfluente hypergeometrische Funktion (Berlin, 1953).

3. E. D. Cashwell, The asymptotic solutions of an ordinary differential equation in which the coefficient of the parameter is singular, Pacific J. Math., 1 (1951), 337-352.

4. R. L. Evans, Solution of linear ordinary differential equations containing a parameter, Proc. Amer. Math. Soc. 4 (1953), 92-94.

5. R. E. Langer, The asymptotic solutions of ordinary linear differential equations, etc., Trans. Amer. Math. Soc., 67 (1949), 461-490.

6. - On the asymptotic solutions of ordinary differential equations, etc., Trans. Amer. Math., Soc., 87 (1935), 397, et seq.

Purdue University

Institute for Fluid Dynamics and Applied Mathematics

University of Maryland 